\title{
The Application of Optoelectronic Elements to Control the Sowing Process
}

\author{
Lukasz Gierz ${ }^{1, *}$ and Włodzimierz Keska ${ }^{1}$ \\ ${ }^{1}$ Poznan University of Technology, Faculty of Transport Engineering, Poland,
}

\begin{abstract}
The article is an overview of scientific and patent literature as well as solutions available on the world market of electronic equipment for seed drills. Recently Dickey-John and Väderstad, which offers the SeedEye system, have been leading companies in this branch. Photoelectric sensors, which do not interfere with the seed flow, are often used to count seeds and control the correctness of sowing. The article describes and presents the results of simulation and laboratory tests of two original innovative concepts of a photoelectric sensor: 1 - with an infrared diode and a centrifugal seed stream concentrator, 2 - laser multi-reflection sensor
\end{abstract}

\section{Introduction}

There is an increasing need for precision of seed drills used in agriculture. The precision of a seed drill is determined by the following parameters: keeping the assumed sowing norm, i.e. the seeding density per area unit, equal distance between seeds and optimal sowing depth. Like in any other field, the precision of operation should be controlled. In practice, the sowing norm is set permanently during the calibration test before sowing. The longitudinal unevenness of sowing is caused by the way the sowing unit works and possible disturbances in its operation, such as uneven rotation of the sowing shaft or clogging of the seed tube. The latter causes very large losses due to leaving areas of land unseeded. It is very difficult to detect clogging, especially in seed drills with large working width. In this case, grain flow sensors installed on seed tubes, may be particularly helpful. In mechanical and pneumatic seed drills, which are commonly used, it is usually impossible to control the sowing process in individual seed tubes, although researchers are working on the development of new methods [1-7]. There have been scientific, patent and specialist publications giving examples of solutions based on optical sensors [8]. The detectability of various sensors was tested in earlier studies [9-11]. A linear image intensifier could be used as an alternative, but this solution is still expensive and requires complex software for image analysis [12]. Thanks to high-precision sensors it is possible to control the current sowing norm and make automatic changes according to the sowing map. While it is very easy to count the number of seeds sown by precision seed drills, it is difficult to do it in inline seed drills.

\footnotetext{
* Corresponding author: lukasz.gierz@.put.poznan.pl
} 


\section{Material and methods}

\subsection{Overview of seed sensors in seed drills}

The following three types of sensors are used in agricultural practice for the detection of seeds in seed tubes: photoelectric, capacitive and piezoelectric sensors. Photoelectric sensors are the most common - seeds passing through the sensor interrupt the beam of light running from the source of light (usually LED diodes) to the photoelement (usually a phototransistor or photoresistor). Advanced solutions, such as those patented by the DJ company, use a rather complex analogue electronic system which compensates for the weakening of photoelement lighting caused by dirt and which distinguishes between seeds passing close to one another. The latest solutions presented by the Väderstad company (see Fig. 1) and awarded at agricultural machinery fairs use optical barriers in the form of a palisade of single rays. According to the manufacturer, the detectability of seeds by these sensors is as high as $98 \%$.

a)

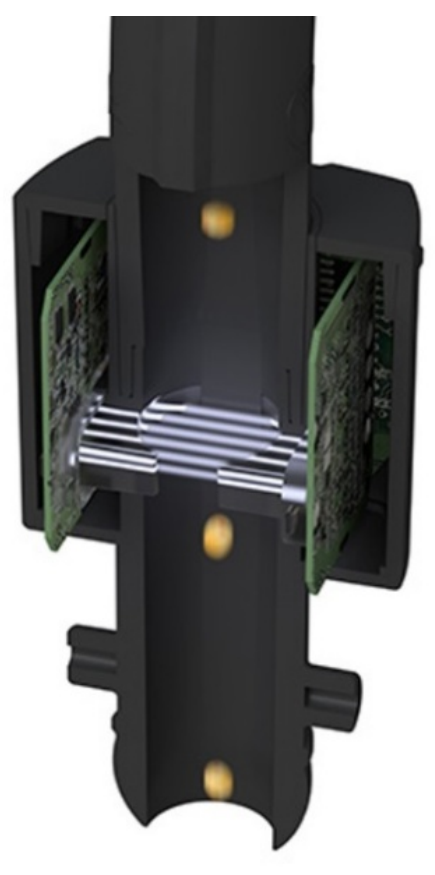


b)

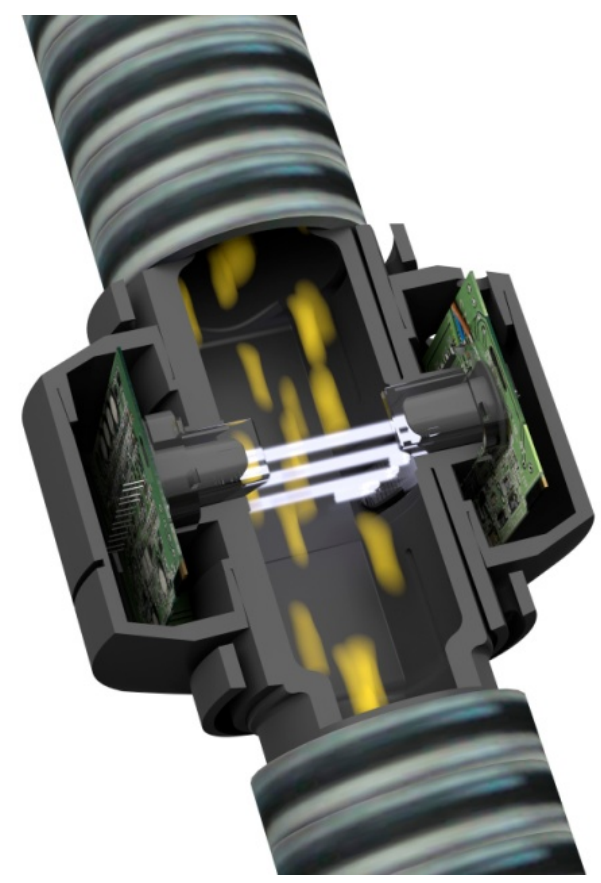

Fig. 1. A Väderstad photoelectric sensor: a) round caryopses, b) ellipsoidal caryopses [8]

Capacitive sensors detect seeds passing between two electrodes, which make a flat air capacitor. They do not disturb the flow of seeds but they have relatively low resolution and are used rather rarely. They also require sensitive and expensive analogue electronic circuits to form standard pulses. Piezoelectric sensors detect seeds that hit the surface of the sensor. It is an advantage that they are not sensitive to dirt and dust. As results from the principle of their operation, they interfere with the flow of seeds, which can be both an advantage and a disadvantage.

\subsection{Original constructional designs of optical seed sensors}

In order to improve the quality of photoelectric sensors and cut the costs of their production scientists from the Poznań University of Technology, Poland, prepared two new designs. The first design is chiefly useful for pneumatic seed drills, where seeds are transported in an air stream. The designers concentrated on solving the problems of bypassing optical barriers, i.e. the light beam, by seeds disseminated along the width of the seed tube. For this purpose, a photoelectric sensor consisting of a single photodiode and phototransistor was placed near the outer surface of the elbow of the rectangular seed tube. As the centrifugal force makes seeds move along the surface of the elbow, almost all of them may cut the ray of the optical gate. This solution is shown in Fig. 2. By using a single narrow beam, which can be completely obscured by passing seeds, it is possible to simplify the electronic system, which should generate a rectangular pulse at the output. Such pulses can be easily counted by electronic counters located in each microcontroller. 


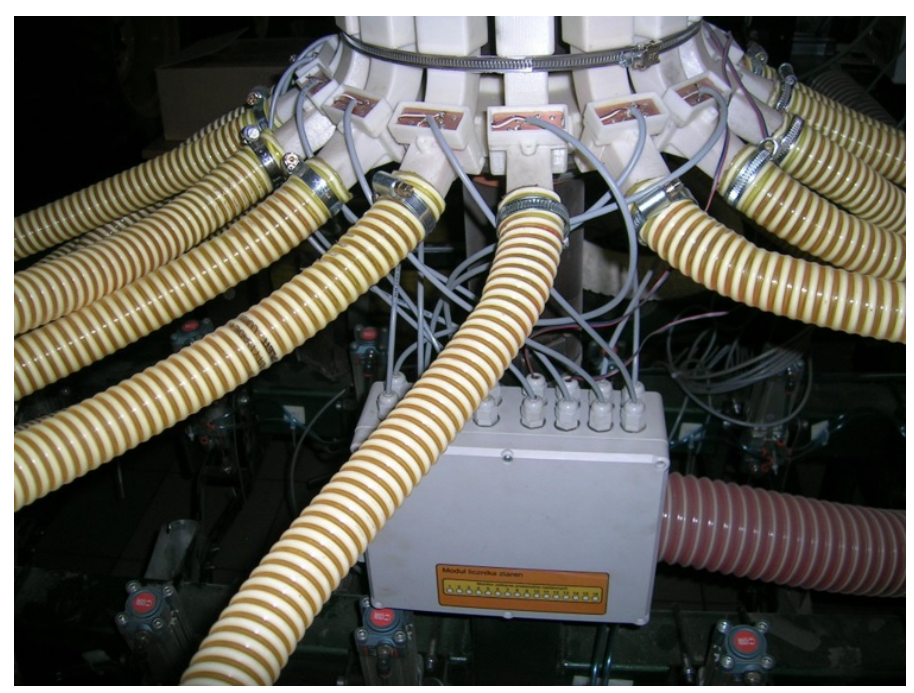

Fig. 2. An original design of the distribution head integrated with seed sensors in a pneumatic seed drill

In the other design, a light beam generated by a semiconductor laser is repeatedly reflected by two mirrors facing each other. Thus, the entire cross-sectional area of the seed tube is controlled by a single beam of light. This sensor immediately sends a binary signal that is easy to process by digital systems. Currently this solution is being tested in a laboratory.

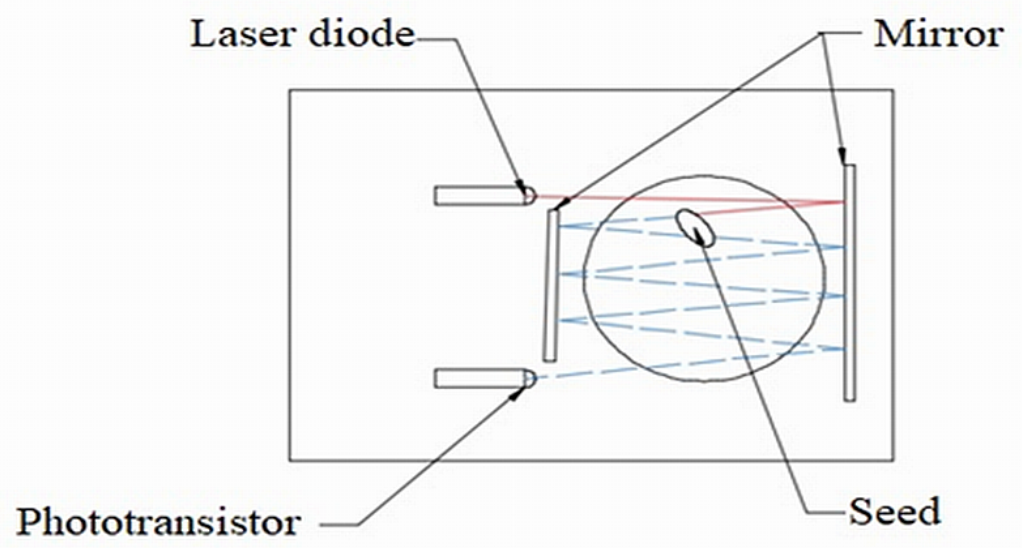

Fig.3. A scheme of a laser sensor with multiple light beam reflection

In this case the main problem is that it is very likely that several seeds may go through the optical gate simultaneously. As empirical and simulation studies showed, the distribution of distances between seeds flying in the seed tube has exponential nature, which means that the distance close to zero is the most probable. Therefore, it is impossible to see all seeds in this way. The greater the seed flow rate in the seed tube is, the better the seed discrimination is. Therefore, a special distribution head was designed for a pneumatic seed drill to avoid diversified seed flow rates in tubes and to ensure identical conditions of the flow through the sensors. Thus, the result of counting can be corrected mathematically. The same solution is applied in some patented seed flow sensors. 


\section{Results}

Detailed simulation tests of the sowing process and movement of seeds through seed tubes were conducted to check the theoretical possibility of detection of individual seeds by various types of optical barriers. The simulation consisted in generating a shadow of flying seeds, which were evenly dispersed in the lumen of the cross section of the seed tube and arranged longitudinally according to the exponential distribution. They generated a series of rectangular pulses in an optical gate placed at different points of the seed tube. Later, the influence of the seed size, the sowing norm, the cross-sectional area of the seed tube and the flow rate in the seed tube on the degree of detectability of these seeds was examined. Figures 4 and 5 show a view of the interface of the computer program used in the test. The following values of parameters were assumed: sowing density - 400 caryopses $/ \mathrm{m}^{2}$, sowing velocity $-2 \mathrm{~m} / \mathrm{s}$, thousand kernel weight - $20 \mathrm{~g}$, spherical shape of seeds (shape factor - 1), seed tube width $-5 \mathrm{~mm}$, seed tube height $-30 \mathrm{~mm}$, filming step $-0.1 \mathrm{~ms}$, averaging time $1,000 \mathrm{~ms}$, number of repetitions - 10 , number of sensors -3 , and measurement section length $-2 \mathrm{~m}$. The ability to distinguish individual seeds in the shadow image obtained on the image sensor matrix was examined in the same way. Figure 4 shows selected results of the test for the first variant of the sensor with an infrared diode and a centrifugal seed stream concentrator, a transport air velocity of $1 \mathrm{~m} / \mathrm{s}$ and dimensions of the field of observation $1 \times 1 \mathrm{~mm}$. Figure 5 shows the results for the second variant of the multireflection laser sensor, at a transport air velocity of $12 \mathrm{~m} / \mathrm{s}$ and dimensions of the field of observation $5 \times 3 \mathrm{~mm}$. As the test results show, at the lower air velocity of $1 \mathrm{~m} / \mathrm{s}$ and small area of the field of observation $1 \times 1 \mathrm{~mm}$ shadows of caryopses overlapped very often (see the blue bar in Fig. 4) and made counting difficult - in this case $53.94 \%$ and $78.84 \%$ of the caryopses were counted. At the air velocity of $12 \mathrm{~m} / \mathrm{s}$ and the area of the field of observation $5 \times 3 \mathrm{~mm}$, the shadows of caryopses were away from each other (see the blue bar in Fig. 5), which facilitated the counting process $-95.44 \%$ and $97.93 \%$ of the caryopses were detected.

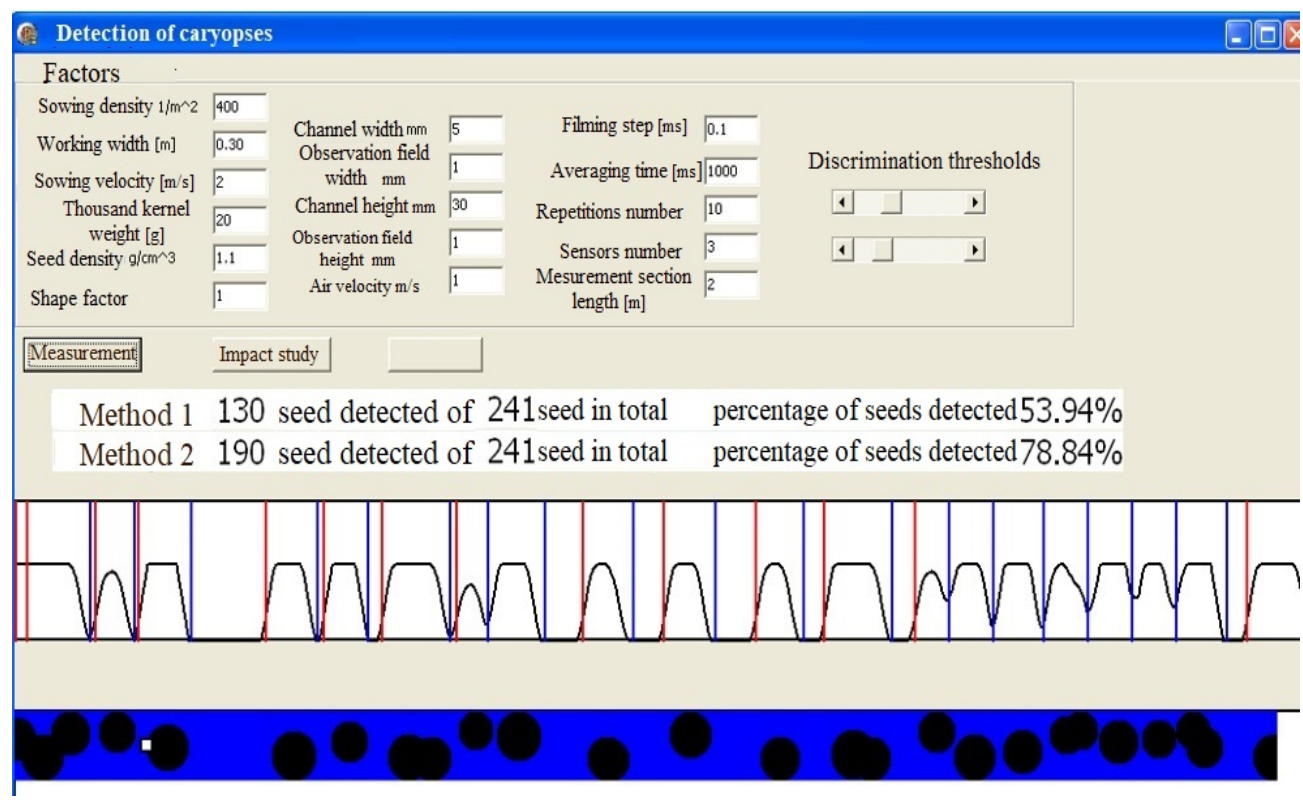

Fig. 4. The results of simulation of the seed counting process at a low air flow rate in the pneumatic 
conduit

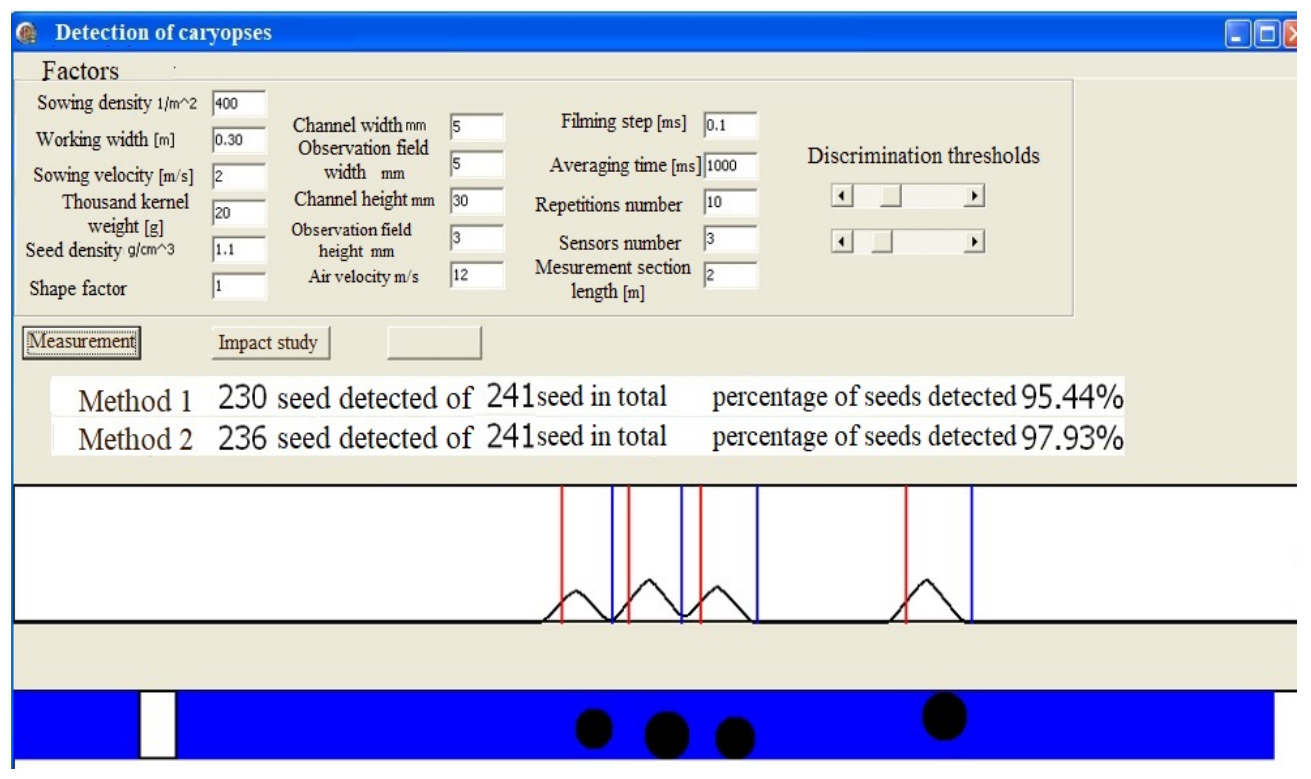

Fig. 5. The results of simulation of the seed counting process at a high air flow rate in the pneumatic conduit

\section{Conclusions}

As earlier studies theoretically and empirically proved $[6,13]$, the accumulation of random factors results in exponential distribution of the distance between seeds sown by inline seed drills. In this distribution distances between seeds are most likely to be close to zero. For this reason, it is very difficult to count seeds during sowing, because optical sensors cannot discriminate seeds flying too close to one another. There are better results of counting in pneumatic seed drills, in which seeds fly at higher speeds in the seed channel (e.g. Amazone EDX) [14] and at greater distances from each other. The optical sensors built at the Poznań University of Technology are based on a single beam of light and simple digital systems. They detect clogging easily and, to some extent, they control the sowing norm and symmetric distribution of the seed stream in the distribution head, having corrected the result of counting mathematically, using the probability theory. Apart from the research on optical sensors, there are also studies on piezoelectric, impact sensors made from special organic materials with piezoelectric properties. They are the subject of other studies in this field under the LIDER VIII project co-financed by the National Centre for Research and Development.

\section{Acknowledgements}

The research was supported by the National Centre for Research and Development under the LIDER VIII project No. LIDER / 24/0137 / L-8/16 / NCBIR / 2017.

\section{References}


1. E. Çakir, I. Aygün, A. Yazgi, Turkish Journal of Agriculture and Forestry, 40, 874881, (2016).

2. Ł. Gierz, Journal of Research and Applications in Agricultural Engineering, vol.62/1, 27-30 Poznań (2017).

3. D. Karayel, M. Wiesehoff, A. Ozmerzi, J. Muller, Computers and Electronics in Agriculture 50 (2006).

4. Y. Lan , M. F. Kocher, A. Smith. Journal of Agricultural Engineering Research, 72, 119-127, (1999).

5. A. Ózmerzi, D. Karayel, M. Topakci, Biosystems Engineering, 82 2, 227-230, (2002).

6. W. Kęska, Ł. Gierz., Mathematical modeling and computer simulation of sowing, Proceedings of 69 International Conference on Agricultural Engineering Land TECHNIK AgEng 2011 nt. "Solutions for Intelligent and Sustainable Farming", VDI_Berichte Nr 2124.2011, Hannover, Germany, 2011, 459-464, (2011).

7. V. J. Kumar, C. Divaker, C. Durairaj, .J. agric. Engng Res. 75, 81-95, (2000).

8. https://www.vaderstad.com/pl/o-nas/aktualnoci-i-prasa/archiwumwiadomoci/2015/international/nowe-moliwoci-z-sensorem-seedeye/

9. Ł. Gierz, W. Kęska, Journal of Research and Applications in Agricultural Engineering, 562 , (2011).

10. Ł. Gierz, Journal of Research and Applications in Agricultural Engineering, 60 1, 11 $13,(2015)$

11. H. Karimi., H. Navid, A. Mahmoudi, Spanish Journal of Agricultural Research, 13 1, (2015).

12. K. Deguchi, Measurement, Volume 4, Issue 4 (1986)

13. W. Kęska, Ł. Gierz. Modeling and simulation of the seed sowing process with pneumatic seed drills. Materials of the VII Symposium on "Modeling and computer simulation in technology", Lodz, 22-23.04.2010, 73-78 (2010).

14. http://www.amazone.pl/1262.asp?for_lang=1 